\title{
Caracterización Productiva de los Sistemas Ganaderos y el Manejo del Recurso Pastizal en el Cantón Joya de los Sachas
}

\author{
Raúl Lorenzo González Marcillo, Ing. Zootecnista MSc. \\ Robinson Ulices Carrasco Carrasco, Ing. Zootecnista MSc \\ Fabián Segundo Guamán Quinche, Médico Veterinario y \\ Zootecnista MSc
}

Universidad Escuela Superior Politécnica de Chimborazo Extensión Norte Amazónica de la República del Ecuador

Doi: 10.19044/esj.2019.v15n18p355 URL:http://dx.doi.org/10.19044/esj.2019.v15n18p355

\section{Resumen}

El objetivo del presente trabajo fue caracterizar los sistemas ganaderos en el Cantón Joya de los Sacha, Provincia de Orellana de la Región Amazónica del Ecuador, se realizó una valoración de los indicadores técnicos-productivos a 50 fincas ganaderas seleccionadas al azar y ubicadas en un radio de $61 \mathrm{~km}$ de la sede del Centro académico de la Extensión Norte Amazónica de la Escuela Superior Politécnica de Chimborazo. Los datos fueron obtenidos mediante una encuesta aplicada a los propietarios de las fincas referida al recurso pastizal, población animal, infraestructura y equipo, manejo de la alimentación y destino de la producción. El análisis estadístico fue descriptivo. Las fincas están orientadas al doble propósito (65\%), con potreros de tamaño variables, siendo el promedio 16 ha, donde prevalece el pastoreo rotacional continuo $(95 \%)$. El promedio de la superficie con pastura introducidas es de 8 ha. Los valores de utilización del pasto, tanto vaca/ha fueron 5,6 y 0,2, respectivamente, para la superficie con pasto introducido. Las explotaciones ganaderas en el Cantón Joya de los Sachas se caracterizaron por ser intensivo, con pequeñas áreas de pasto introducido y con poca inversión técnica-productiva.

Palabras clave: Caracterización, pastura, fincas, bovino de doble propósito 


\title{
Productive Characterization of Livestock Systems and Pasture Management in the Canton Joya de los Sachas
}

\author{
Raúl Lorenzo González Marcillo, Ing. Zootecnista MSc. \\ Robinson Ulices Carrasco Carrasco, Ing. Zootecnista MSc \\ Fabián Segundo Guamán Quinche, Médico Veterinario y \\ Zootecnista MSc
}

Universidad Escuela Superior Politécnica de Chimborazo Extensión Norte Amazónica de la República del Ecuador

\begin{abstract}
The objective of the present work was to characterize the livestock systems in the Canton of Joya de los Sachas, Orellana Province of the Amazonian Region of Ecuador, an assessment of the technical-productive indicators was made to 50 livestock farms selected at random and located within a radius of $61 \mathrm{~km}$ of the headquarters of the Academic Center of the North Amazonian Extension of the Higher Polytechnic School of Chimborazo. The data was obtained by means of a survey applied to the owners of the farms related to the grassland resource, animal population, infrastructure and equipment, feeding management and production destination. The statistical analysis was descriptive. The farms are oriented to the double purpose $(65 \%)$, with paddocks of variable size, the average being 16 ha, where continuous rotational grazing prevails (95\%). The average surface area with introduced pasture is 8 ha. The values of grass utilization, both cow / ha were 5.6 and 0.2 , respectively, for the area with introduced pasture. Livestock farms in the Canton of Joya de los Sachas were characterized for being intensive, with small areas of pasture introduced and with little technical-productive investment.
\end{abstract}

Keywords: Characterization, pasture, farms, cattle

\section{Introduction}

Los sistemas de ganadería representan el principal medio de vida y la base de la seguridad alimentaria de casi mil millones de personas en el mundo FAO, (2009). Por otro lado, comprenden alrededor del 26\% de la superficie terrestre y en algunos países las tierras empleadas para el desarrollo de esta 
actividad contribuye al proceso de deforestación de millones de hectáreas Steinfeld et al., (2006).

En sur américa ha causado la transformación de aproximadamente 190,7 millones de hectáreas de bosques a ganadería en muchas regiones Zapata et al., (2007). El avance de la ganadería extensiva en la región es considerada como una actividad con impactos negativos al medio ambiente por el cambio de uso de bosque a pastura y la degradación de los ecosistemas Villacis et al., (2003).

En un sistema ocurren un sinnúmero de fenómenos complejos que requieren una evaluación de punto de vista holístico, es decir, tener un conocimiento de todo nos permite conocer mejor cada uno de los componentes del sistema al igual que sus relaciones entre ellos.

Una herramienta utilizada para evaluación de sistema, lo constituye la caracterización, la cual según Bolaños, (1999) no es más que la descripción de las características principales y múltiples interrelacionadas de las organizaciones.

En este escenario a la ganadería se le considera como una de las actividades agropecuarias que causa mayor impacto negativo sobre el entorno amazónico ecuatoriano asociándose a la deforestación, compactación de tierras por el pisoteo, erosión, perdida de la fertilidad de los suelos, reducción de la biodiversidad y contaminación de las corrientes de agua INIAP, (2010) y Murgueitio e Ibrahim, (2004).

Entre las características que se pueden evaluar en los sistemas ganaderos se encuentran los indicadores técnicos-productivos, que permiten observar las características de manejo del pastizal, recursos de equipos e infraestructura, recursos animales y recursos alimenticios. En tal sentido, el objetivo de este trabajo es la caracterización técnica-productiva de los sistemas ganaderos del Cantón Joya de los Sachas de la provincia de Orellana de la Región Amazónica relacionados a los indicadores sobre manejo de pasturas.

\section{Materiales y Métodos}

Se utilizó una base de datos obtenidas de un estudio de 50 fincas de pequeños y medianos ganaderos del Cantón Joya de los Sachas que se ubica en las coordenadas S $0^{\circ} .30^{\prime} 014^{\prime \prime}$ y $\mathrm{O} 76^{\circ} 85^{\prime} 75^{\prime \prime}$ en un radio de $61 \mathrm{~km}$, alrededor de la sede de la Extensión Norte Amazónica (ENA) de la Escuela Superior Politécnica de Chimborazo (ESPOCH) de la Provincia de Orellana, el centro académico se localiza en la ciudad del Cantón Francisco de Orellana entre las coordenadas Latitud $0^{\circ} 28^{\prime} 16^{\prime \prime} \mathrm{S}$ y Longitud 76 59' 12" W, en una zona clasificada como bosque tropical lluvioso, con altura de $275 \mathrm{msnm}$, precipitación anual $2800 \mathrm{~mm}$ y temperatura promedio anual de $26{ }^{\circ} \mathrm{C}$. INAMHI, (2010). Las principales actividades económicas que generan fuentes de trabajo son la ganadería y la agricultura, en la que se destacan los 
cultivos de maíz, arroz, café, cacao y palma africana González \& Pérez, (2018).

Para la obtención de la base de datos se utilizó la encuesta como instrumento de evaluación mediante un muestreo de tipo aleatorio cuya orientación fundamental fue la producción con bovinos conjuntamente con la observación directa de los recursos físicos y procesos claves que caracterizan el manejo de estas explotaciones. El análisis estadístico de los indicadores técnicos fue mediante estadísticas descriptiva (promedio, máximo, mínimo e intervalos de frecuencia), utilizando una hoja de cálculo para el procesamiento de los datos Microsoft Excel, (2013).

\section{Resultados y Discusión}

Como se observa en el Cuadro 1, la disposición productiva ganadera es diversa orientándose principalmente a la explotación de doble propósito (65\%), seguida por la explotación de carne y de leche (24 y 11\%, respectivamente), debido a su elevado número de vacas reproductivas, a las cuales se ordeñan para producir queso artesanal y venta de leche cruda. El sistema de pastoreo predominante es rotacional continuo (95\%) con poca inversión en subdivisión de potreros, debido a sus pequeño tamaño (1,4 ha), los cuales poseen una baja superficie de pastura cultivada de mediano valor nutritivo, sin suplementación, dirigida al engorde de novillos por ser básicamente energética. Al respecto Alfaro, (2005) indico que la baja disponibilidad de forrajes en cantidad y calidad y la no suplementación mineral tienen efectos sobre la producción y reproducción de los rebaños. 
Tabla 1. Indicadores técnico productivos de los sistemas ganaderos Cantón Joya de los Sachas.

\begin{tabular}{|c|c|c|c|c|c|c|c|c|c|c|c|c|c|c|c|c|c|}
\hline \multirow{2}{*}{ Indicador técnico } & \multirow{2}{*}{ Prom } & \multirow{2}{*}{ Mín } & \multirow{2}{*}{ Máx } & \multicolumn{14}{|c|}{ Intervalo } \\
\hline & & & & Frec & $\%$ & Frec & $\%$ & Frec & $\%$ & Frec & $\%$ & Frec & $\%$ & Frec & $\%$ & Frec & $\%$ \\
\hline & & & & $<21$ & & $21-37$ & & $37-53$ & & 53-69 & & $69-85$ & & $85-101$ & & $>120$ & \\
\hline \multirow[t]{2}{*}{$\begin{array}{c}\text { Tamaño de la finca, } \\
\text { ha }\end{array}$} & 16 & 5 & 120 & 20 & 40 & 11 & 22 & 10 & 20 & 3 & 6 & 1 & 2 & 3 & 6 & 2 & 4 \\
\hline & & & & $<10$ & & $11-19$ & & $20-28$ & & 29.37 & & $38-46$ & & $47-55$ & & $>64$ & \\
\hline \multirow[t]{2}{*}{$\begin{array}{c}\text { Superficie con } \\
\text { pasto, ha }\end{array}$} & 8 & 2 & 56 & 22 & 44 & 14 & 28 & 8 & 16 & 1 & 2 & 2 & 4 & 2 & 4 & 1 & 2 \\
\hline & & & & $<2,4$ & & $2,5-3,9$ & & $4-5,4$ & & $5,5-6,9$ & & $7-8,4$ & & $8,5-9,9$ & & $>11,4$ & \\
\hline \multirow[t]{2}{*}{$\begin{array}{c}\text { Tamaño de los } \\
\text { potreros, ha }\end{array}$} & 1,4 & 1 & 20 & 25 & 50 & 9 & 18 & 6 & 12 & 2 & 4 & 2 & 4 & 4 & 8 & 2 & 4 \\
\hline & & & & $<7$ & & $7-12,6$ & & $12,6-18,2$ & & $18,2-23,8$ & & $23,8-29,4$ & & $29,4-35$ & & $>40,6$ & \\
\hline \multirow[t]{2}{*}{ Hectárea por vaca } & 5,6 & 1,4 & 40,6 & 21 & 42 & 13 & 26 & 9 & 18 & 2 & 4 & 0 & 0 & 4 & 8 & 1 & 2 \\
\hline & & & & $<6$ & & $6-11$ & & $11-15$ & & $15-20$ & & $20-25$ & & $25-30$ & & $>35$ & \\
\hline \multirow[t]{2}{*}{$\begin{array}{c}\text { Hectárea de pasto } \\
\text { cultivado por vaca } \\
\text { masa }\end{array}$} & 4,8 & 1 & 35 & 21 & 42 & 13 & 26 & 9 & 18 & 2 & 4 & 0 & 0 & 4 & 8 & 1 & 2 \\
\hline & & & & $<0,6$ & & $0,6-0,8$ & & $0,8-1$ & & $1-1,2$ & & $1,2-1,4$ & & $1,4-1,6$ & & $>1,8$ & \\
\hline $\begin{array}{c}\text { Carga animal total } \\
\text { real, UBA }\end{array}$ & 0,2 & 0,4 & 1,8 & 20 & 40 & 13 & 26 & 1 & 2 & 5 & 10 & 5 & 10 & 1 & 2 & 5 & 10 \\
\hline $\begin{array}{c}\text { Hectárea por } \\
\text { equivalente hombre }\end{array}$ & 8 & 2 & 58 & & & & & & & & & & & & & & \\
\hline $\begin{array}{c}\text { Fincas que } \\
\text { fertilizan, } \% \\
\end{array}$ & & $\begin{array}{c}\text { SI } \\
\text { NO } \\
\end{array}$ & $\begin{array}{c}1 \\
99 \\
\end{array}$ & & & & & & & & & & & & & & \\
\hline $\begin{array}{c}\text { Fincas que controlan } \\
\text { malezas, } \%\end{array}$ & & $\begin{array}{c}\text { SI } \\
\text { NO }\end{array}$ & $\begin{array}{l}90 \\
10 \\
\end{array}$ & & & & & & & & & & & & & & \\
\hline $\begin{array}{l}\text { Tipo de explotación } \\
\text { predominante, } \%\end{array}$ & Doble & $\begin{array}{l}\text { oropósito } \\
\text { Carne } \\
\text { Leche }\end{array}$ & $\begin{array}{r}65 \\
\\
\end{array}$ & & & & & & & & & & & & & & \\
\hline
\end{tabular}


Las explotaciones de acuerdo a su superficie total se encuentran entre medianas y pequeñas, debido a que el $62 \%$ de las fincas son menores a $37 \mathrm{ha}$, con un promedio total para las fincas encuestadas de 16 ha. Un $72 \%$ de las fincas tienen menos de 36 ha con pastizales introducidos con un promedio de 8 ha. Páez et al., (2003) encontraron que las fincas están principalmente ubicadas entre pequeñas y medianas con una superficie total promedio de 136,5 ha y una superficie promedio con pasto de 116,4 ha.

En el manejo de los pastizales, la superficie que ocupa cada unidad animal expresada en vaca/ha. fue en promedio 5,6 para el total de la superficie de la finca, y 4,8 vaca/ha. para la superficie con pasto cultivado en la finca para una carga animal de 0,2 UBA/ha. Estas cifras indican que se favorece el sobrepastoreo lo que compromete la permanencia de los pastizales. El número de fincas que manifestaron realizar labores de fertilización y control de maleza fue de $1 \%$ y $90 \%$, respectivamente.

\section{Conclusiones}

La mayoría de las explotaciones ganaderas que se encuentran en el Cantón Sacha manifiestan ser de tipo doble propósito, debido a que realizan actividades económicas, unas con la venta artesanal de queso y otras con las ventas esporádicas de reses principalmente secas. Este comportamiento permite determinar que en la realidad, estas explotaciones doble propósito no son más que una consecuencia de las explotaciones de carne o de leche, al ser estas últimas poco eficientes en su actividad económica.

Las explotaciones se caracterizan por tener pequeñas extensiones de tierras compuestas principalmente por cultivos agrícolas y pasturas introducidas con poca inversión en subdivisión de potreros. Las pasturas introducidas se manejan bajo pastoreo rotacional continuo con carga animal muy baja, lo cual, generan problemas por poca disponibilidad de forrajes provocando la desaparición de las pasturas.

\section{References:}

1. Alfaro C.; Rodríguez I.; Guevara E. ; Godoy S.; Navarro L.; Ramírez M.; Connell J.; Tirado H. (2005). Proyecto de investigación para el oriente del país: Mejoramiento de la sostenibilidad y competitividad de los sistemas de producción bovinos. Resúmenes Jornadas Técnicas INIA Falcón. INIA CIAE Falcón. Coro, Falcón.

2. Bolaños O. (1999). Caracterización y tipificación de organizaciones de productores y productoras. Costa Rica. Unidad de Planificación Estratégica. Ministerio de Agricultura y Ganadería

3. FAO (2009). El estado mundial de la agricultura y la alimentación. Roma-Italia. Viale delle Terme di Caracalla, 00153. 
4. González R. \& Perez J. (2018). Percepciones y Caracterización De Pastizales En Los Cantones Joya De Los Sachas Y Francisco De Orellana. Orellana-Ecuador. ENA-ESPOCH

5. INIAP (2010). Mejoramiento y recuperación de la investigación, soberanía, seguridad alimentaria y desarrollo agropecuario sostenible en la amazonia ecuatoriana. Disponible en: http//www,iniap,gob,ec.

6. INAMHI (2010). Anuario meteorológico. Quito- Ecuador. Versión preliminar 50

7. Murgueitio E. \& Ibrahim M. (2004). Ganadería y medio ambiente. XII Congreso Venezolano de Producción Animal. p. 187-202

8. Steinfeld H.; Gerber P.; Wassenaar T.; Castel V.; Rosales M.; de Haan C., (2006). Livestock's long shadow. Roma-Italia,.Information división FAO

9. Microsoft Excel (2013). Versión 10.0. Microsoft Corporation. USA:

10. Páez L.; Linares T.; Sayago W.; Pacheco R. (2003). Caracterización estructural y funcional de fincas ganaderas de doble propósito en el Municipio Páez del estado Apure, Venezuela. Zootecnia Trop., 21(3):301-323.

11. VILLACÍS J.; Harvey C.; Ibrahim M.; Villanueva C. (2003). Relaciones entre la cobertura arbórea y el nivel de intensificación de fincas ganaderas en Río Frío, Costa Rica. Agroforesteria en las Américas, 10 (39-40), 17-23 ISSN: 1022-7482.

12. Zapata A.; Mejía C.; Zuluaga A.; Ibrahim M. (2007). Efecto del pago por servicios ambientales en la adopción de sistemas silvopastoriles en paisajes ganaderos de la cuenca media del Río La Vieja. Colombia. Costa Rica. Revista Agroforesteria en las Américas No. 45 